\title{
Frequency of Expression of PD-1 and PD-L1 In Head And Neck Squamous Cell Carcinoma And their Association With Nodal Metastasis: A Cross-Sectional Study
}

\author{
Iqraa Shakeel Malik ${ }^{1 *}$, Muhammad Asif ${ }^{1}$, Namrah Bashir ${ }^{1}$, Nighat Ara ${ }^{2}$, Farhat \\ Rashid $^{3}$, Hafeez Ud Din ${ }^{3}$, Numrah Shakeel Malik ${ }^{4}$, Aimen Bashir ${ }^{5}$
}

\begin{abstract}
Objective: The objective of this study was to determine the Immunohistochemical expression of PD- 1, PD-L1 and its association with nodal metastasis in head and neck squamous cell carcinoma. Materials and Methods: A total of 66 cases were studied at Oral pathology/Histopathology, Armed forces Institute of Pathology (AFIP), Rawalpindi. The tissue sampling and processing yielded the formalin fixed, paraffin wax blocks. These blocks were cut into the thin sections ( 5 microns) by the microtome and then were mounted on the glass slides. This was followed by the routine H\&E staining and then IHC staining (PD-1 and PD-L1) of these tissues mounted slides. For each case a definitive histological diagnosis was made. The two types of variables were analyzed. For qualitative variables frequencies and percentages were calculated whereas for quantitative variables means and standard deviations were analyzed. The Chi-square test then was applied to evaluate the significant difference and $p$-value of $\leq 0.05$ was taken significant. Results: This study was conducted at Histology department, Armed Forces Institute of Pathology, Rawalpindi over a period of one year from June 2019 to June 2020. It revealed male and female patients with 66.7\% (44 Cases) and 33.3\% (22cases) percentages respectively. The mean age was found to be $59.53 \pm 13.637$ (mean \pm SD) and the mandible (37.9\%) was the most commonly affected site. In total of 66 cases, $48(72.7 \%)$ cases with metastasis of lymph nodes were presented. Out of 66 cases ,47 (71.2\%) cases showed positive expression of PD-1 in the TILs seen among the primary tumor of the specimens, whereas, $40(60.6 \%)$ cases showed positive PD-L1 expression in tumor cells of primary tumor.In comparison, as out of 66 cases ,48 (72.5\%) cases showed lymph nodes metastasis, out of which 45 showed positive expression for PD-1 and 25 out of those 48 cases showed positive expression for PD-L1 in metastatic lymph nodes. The p-value turned to be significant for PD-1 IHC expression and PD-L1 IHC expression in the primary tumorand metastatic lymph nodes. Conclusion: A significant correlation was inferred among IHC expression of PD-1 and PD-L1 with lymph nodes metastasis. Accurate evaluation, analysis and precise management with aid of IHC markers results in initial and timely diagnosis and favorable treatment outcomes helping in the evaluation of disease course at preliminary diagnosis on incisional biopsies.
\end{abstract}

Keywords: Biomarker- Cd274 protein- immunohistochemistry- oral and maxillofacial pathology- oral neoplasms

Asian Pac J Cancer Prev, 23 (2), 467-473

\section{Introduction}

The cancers of Head and Neck region are reported to be at the sixth level in the list of commonest cancers in the world, with commonest histological type as squamous cell carcinoma (Wang et al., 2015). In spite of the extensive and advanced research area, the significant mortality and morbidity rates are seen in HNSCC (Patil et al., 2014). The most major risk factors of OSCC include mainly tobacco (smoked, smokeless), alcohol, poor nutrition, high risk types of human papillomaviruses and poor oral hygiene (Zeng et al., 2020). By immunoediting, immune system playa a dual part in tumor (Teixidóet al., 2018). The innate and adaptive immune responses play major role in constraining the tumor growth and destroying the cancer cells in immunosurveillance. Whereas, the tumors can also enter into an 'escape' phase that leads to the characteristic immune suppression state. It is caused by recruitment of immunosuppressive cells (Schreiberet al., 2011). The tumor cells evade and evolve the immune

${ }^{1}$ Department of Histopathology, Armed Forces Institute of Pathology, National University of Medical Sciences, Rawalpindi, Pakistan. ${ }^{2}$ Department of Oral Pathology, Army Medical College, Rawalpindi, Pakistan. ${ }^{3}$ Consultant Histopathologist, Armed Forces Institute of Pathology, National University of Medical Sciences, Rawalpindi, Pakistan. ${ }^{4}$ Department of Oral and MaxilloFacial Surgery, FUCD, Fauji Foundation Hospital, DHA 1, Islamabad, Pakistan. ${ }^{5}$ Department of Oncology, Fauji Foundation Hospital, DHA 1, Islamabad, Pakistan.*For Correspondence: malikiqraa@yahoo.com 
system by manipulation of self-immunogenicity and/ or by the expression of immunosuppressive mediators, this marks the basis of the development of advanced immunotherapy(Al Azhar and Aisyi, 2021). The check point controllers comprise of the cytotoxic T-lymphocyteassociated antigen-4 (CTLA-4), programmed cell death-1 (PD-1) and programmed cell death ligand-1 (PD-L1) (Teixidó et al., 2018).

PD-1/Cluster of differentiation 279 is on the surface of cells as a protein that plays a significant part in immune response regulation by down-regulation of immune system and promotion of self-tolerance by suppression of the $\mathrm{T}$ cells inflammation activity. This helps in preventing autoimmune diseases along with preventing the immune system from killing the cancer cells as well (Syn et al., 2017). Increased expression of PD-1 at CD8+ T cells indicates T-cell fatiguee.g. in cancers (Syn et al., 2017).

Programmed cell death-ligand 1 is a $40 \mathrm{kDa}$ type 1 transmembrane protein which plays an significant part at suppression of adaptive immune system (Chemnitz et al., 2004) C.When PD-L 1 binds to PD-1, a repressive signal is transmitted which results in reduction of antigen specific T-Cells in the lymph nodes and apoptosis of T cell (Elmusrati et al., 2021).

In the fields of reconstructive surgery and diagnostic accuracy, studies have showed marked development in the subsistence rate of patients with SCC (Gorsky et al., 2004). Failure to treat squamous cell carcinoma effectively shows lethal results due to its recurrence and distant metastasis. In recent advancements it is believed that the therapeutic failure in treatment of OSCC may be related to malfunctioning of immune system (Maruse et al., 2018).

Further studies have showed that blocking of the PD-L 1/PD-1 checkpoint is being used effectively in treating melanoma and non-small cell lung cancer (Brahmer et al., 2012). However, very few studies have examined the expression and prognostic value of PD- L 1/PD-1 in HNSCC (Lin et al., 2015). The PD-1 and PD-L1-targeted inhibitors show a dynamic part in cancer immunotherapy (Hanna, 2020). The goal of this study is to further insight and check the expression of PD-L1 in the primary tumor cells and PD-1 in TILs and related lymph node metastasis along with some other clinicopathological factors, which in turn will help the clinician and oncologist in many ways, like better delineation of treatment regimens along with the prognosis of the disease. Also, it will help in the correct assessment of disease course at the initial diagnosis on incisional biopsies.

Recently the FDA has approved anti-PD - 1and antiPD - L1 m Abs which targets varied range of the human cancers (Chen and Han, 2015).

\section{Materials and Methods}

\section{EthicalApproval}

IRB approval was given by the Ethical Review Committee AFIP vialetter: MP-ORP18-6/READIRB/19/645

\section{Sample Collection}

This cross-sectional study was carried out on the total of 66 cases of head and necksquamous cell carcinoma collected from the department of Histopathology, AFIP, Rawalpindi, Pakistan over a period of one year from June 2019 to June 2020. Non probability convenience sampling was used to achieve the required sample size including the patients of all ages and both genders. The freshcases of HNSCC irrespective of the grade and differentiation diagnosed on H\&E submittedat Histopathology department were included in the study, whereas the Specimens withthe poorfixation, scarceandscantytissue, patients withpostchemoradiotherapytissuesamples and the necrotictumor were excluded from this study. Of the selected cases, clinical and demographic detail soft hepatients werere corded by the histories provided with the cases. To avoid the confounding factors, the inclusion criteria and the exclusion criteria were followed. The H\&E slides were used to obtain hedefinitive diagnosis which was followed by the IHC staining.

\section{Immunohistochemicalstaining}

The IHC markers were applied by using indirect IHC technique. MonoclonalrabbitantibodytoPD1(CloneEP239,Catalogueno:PR196-6mlRTU;PathnSitu) and Monoclonalrabbitantibody toPD-L1(CloneRBT-PDL1,Catalogueno:BSB2649;BioSB) was used by following the standard protocol of application. The microscopic results were also evaluated and verified by the consultant histopathologist.

\section{Evaluation Of IHC}

The PD-1 andPD-L1 positivecellsweredefinedas Brownstainingofthe Cellmembrane/cytoplasm ofthecells and depending upon this intensity of staining the cases were recorded as positive and negative staining. The controlforPD-1 wasLymphnodeandTonsil with the recorded staining pattern as cytoplasmicand/ormembranousstaining. The controlforPD-L1 wasLymphnode with the recorded staining pattern ascytoplasmicandmembranousstaining.

\section{StatisticalAnalysis}

The data analysis was performed by using the data collection proforma and compiling and analyzing the collected data on the SPSS (version 24.0). Followed by the calculation of frequencies and percentages of the qualitative variables (gender, site, laterality,Differentiation,IHC expression of PD-1 and PD-L1) and means and standard deviations of the quantitative(age,pT,pN) variables. The chi square test was used to compare and analyze the results and access the significance of the difference. The p-value $<0.05$ was set as the significant value.

\section{Results}

A total 66 cases of oral squamous cell carcinoma with corresponding neck dissections were collected In $72.7 \%$ (48) specimens the associated lymph node metastasis was seen. Out of 66 patients 44 (66.7\%) males and $22(33.3 \%)$ females were included which corresponded to the male: female ratio $2: 1$. The mean age distribution was 59.5 years \pm 13.637 (range 34 to 91 years). 
DOI:10.31557/APJCP.2022.23.2.467

Immunohistochemical Expression Of PD-1, PD-L1 And Its Association With Nodal Metastasis

Table 1. Crosstabulation of Expression of PD-1 in TILs in Tumor Proper Sections and Metastasis of Tumori n Lymphnodes

\begin{tabular}{llcccc}
\hline & & \multicolumn{2}{c}{ MetastasisoftumorinLymphnodes } & Total & P-Value \\
& & Seen & Not Seen & & \\
\hline ExpressionofPD-1inTILsintumorpropersections & Positive & $39(81.2 \%)$ & $8(44.4 \%)$ & 47 & $0.003^{*}$ \\
& Negative & $9(18.7 \%)$ & $10(55.5 \%)$ & 19 & \\
Total & & 48 & 18 & 66 & \\
\hline
\end{tabular}

*SignificanceLevel(at5\%, $<<0.05)$

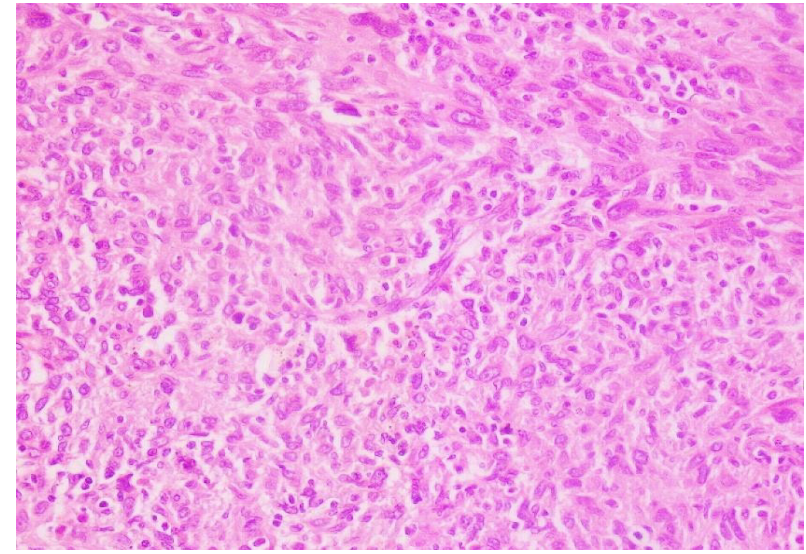

Figure 1. Haemotoxylin and Eosin Stained Poorly Differentiated Oral Squamous Cell Carcinoma Section (Hemi Glossectomy Specimen) (40x).

According to the histological gradin gofhead and neck squamous cell carcinoma the rewere 22 (33.3\%) well differentiated SCC cases, 28 (42.4\%) moderately differentiated SCC cases, 15 (22.7\%)poorly differentiated SCC cases whereas grade of one case couldn't be assessed.

The maximum number of HNSCC cases were seen inmandible $(37.9 \%)$ followed by maxilla $25.8 \%$, buccalmu cosa $13.6 \%$, tongue $10.6 \%$, Palate $4.5 \%$, floor of the mouth $4.5 \%$ and salivary glands $3 \%$. The expression of PD -1 in TIL sand PD- L1 intumorcells was evaluatedin primary tumor sections of the66specimens andtheirexpression wasalsoseenonthe corresponding metastatic lymphnodesin 48 specimens.

\section{PD-1andPD L1expressioninpropersectionsof tumor}

In total of 66 cases, $47(71.2 \%)$ cases showed positive PD - 1 expression in TIL s seen in the primary tumor sections of the specimens, whereas out of 66 cases 40 $(60.6 \%)$ cases showed +ve PD - L1 expression in the effected cells of primary tumor.

\section{PD-1 and PD- L1 expressionin Corresponding Metastatic Lymph Nodes}

In total of 66 cases , 48 (72.5\%) cases showed lymph

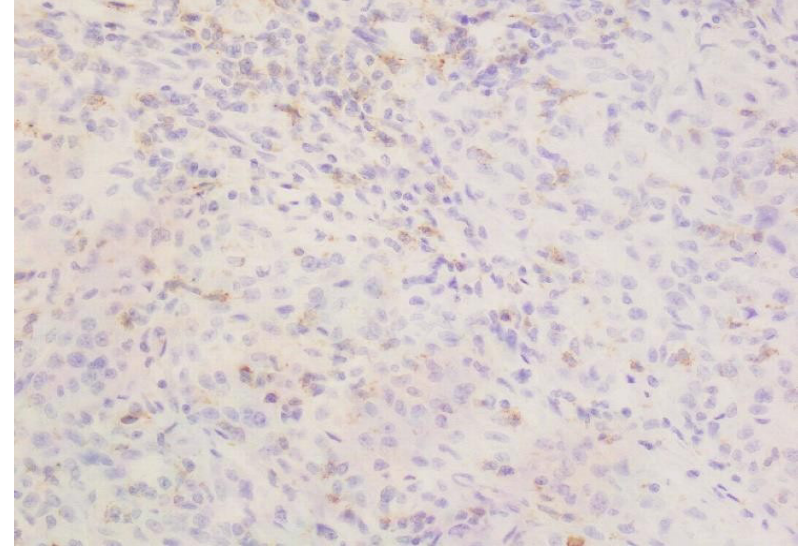

Figure 2. Expression of PD - 1 Showing Focal +ve Membranous Staining (40x).

nodes metastasis, out of which 45 showed positive expression for PD-1 and 25 cases showe dpositive expression for PD-L1 in metastatic lymphnodes.

\section{PD-1expressioninTILs}

The Chi Square test was applied and asignificant association was seen between the PD-1 expression of TILs in primary tumor with lymph nodes metastasis $(p=0.003)$ (represented in Table.1), gender $(p=0.002)$ of

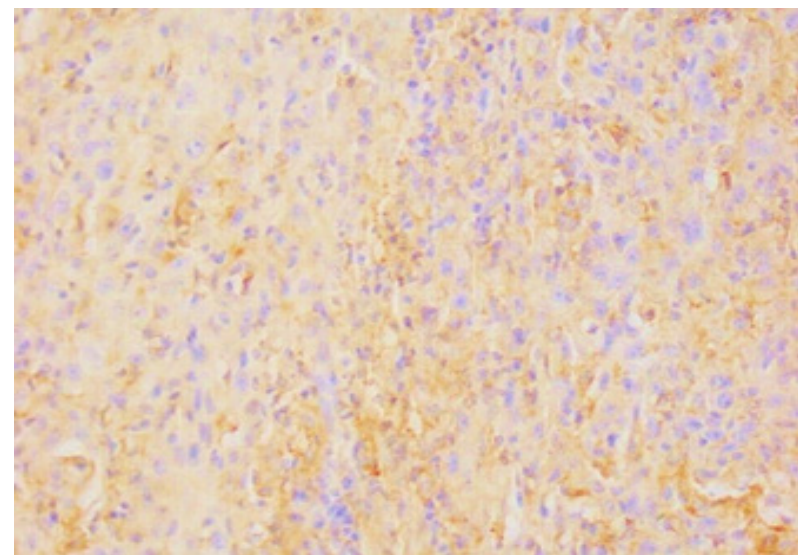

Figure 3. Expression of PD - L1 Showing Positive Membranous Staining (40x)

Table 2. Expression of PD-L1 in Tumor Cells in Tumor Proper Sections *Metastasis of Tumor in Lymphnodes Crosstabulation

\begin{tabular}{lccccc}
\hline & & \multicolumn{2}{c}{ MetastasisoftumorinLymphnodes } & Total & P-value \\
& & Seen & Not Seen & & \\
\hline ExpressionofPD-L1 intumorcellsintumorpropersections & Positive & $33(68.7 \%)$ & $7(38.9 \%)$ & 40 & \\
& Negative & $15(31.25 \%)$ & $11(61.1 \%)$ & 26 & $0.027^{*}$ \\
Total & & 48 & 18 & 66 & \\
\hline
\end{tabular}

*SignificanceLevel (at 5\%, $\mathrm{p}<0.05$ ) 
the patient and the pathological stage of regional lymph nodes $(\mathrm{p}=0.0036)$

Another note worthy correlation seen amongst the expression of PD-1 in TIL sintumorpropersections and expression of PD-1 in the associated metastatic lymph nodes which came out with p-value of 0.003 . However, it showed insignificant results with the pathological staging of primary tumor, age, grades of the tumor, site and laterality of the tumor.

\section{PD-L1expressioninTumorcells}

To assess the association of IHC expression of PD -L1 and associated lymph nodes metastasis, the Chi Square test was applied which showed a significant result $(\mathrm{p}=0.04)$ given in the Table 2. Additionally, a significant association was seen between PD-L1 expression in primary tumor and pathological staging of regional lymph nodes $(p=0.04)$ However, it showed the insignificant results with the pathological staging of primary tumor, age, gender, grades of the tumor, site, laterality of the tumor and the PD-L1 expression in the metastatic lymph nodes.

It was also seen that the PD - L1 expression was lower in the high grade invasive OSCC than that in the low grade invasive OSCC.

\section{Discussion}

Annually the new reported cases of Oral Cancers are about 300,000 cases and approximately, along with OSCC as the commonest oral cancer resulting into high morbidity and mortality rates and showing around 60\% 5 year survival rate (Lenouvel et al., 2020).

For over $90 \%$ of all oral malignancies are OSCC (Goel et al., 2020). Oral Squamous cell carcinoma is reported to be the 6 th commonest malignancy of head and neck(Wang et al., 2015). In spite of the treatment advances a considerable morbidity and mortality is seen in these malignancies (Hanna, 2020; Wu et al., 2016)

This study basically focuses on the latest treatment option for malignancies i.e., immunotherapy. This study targets on the frequency of PD - 1 And PD - L1 expression in HNSCC and associated metastasis in lymph nodes which in turn will direct on the prognosticators and immunotherapy. Very rare studies have analyzed expression and association of PD - 1 and PD - L1 expression in lymph nodes metastasis and primary SCC. Our results would in turn help in the therapeutic decisions and prognosis of HNSCC.

Immunotherapy aims at the normalization and enhancement of anti-tumor immune response of body by killing and inhibiting the cancer cells by immune system activation. PD-1 and PD-L1 immunotherapy show increased efficacy and increase the chance of long-term survival.

Many types of cancers are intractable to the conventional chemotherapy treatment. Checkpoint immunomodulation maintains the inequity between immune surveillance and proliferation of cancer cells which results in tumor survival (Alsaab et al., 2017). PD - 1 and PD - L1 inhibitors (check point blockers), act as a suppressing factor for the tumor through immunomodulation and so are becoming promising therapeutic method with minimum side effects. Recently four inhibitors are accepted by that include PD-1, PDL1, and CTLA-4 inhibitors. In spite of successful results of anti-PD1 therapy it is still used for limited types of cancers (Pardoll, 2012)

The present study aims at the immunohistochemical expression of PD - 1 and PD - L1 evaluation in primary HNSCC and associated metastatic lymph nodes in the patients already treated with conventional surgery.

A total of 66 cases of HNSCC with associated lymph nodes were selected at Histopathology Department of AFIP, Rawalpindi. Out of the 66 cases, 48 (72.7\%) cases showed metastasis in corresponding lymph nodes whereas in a study conducted in Austria in 2018, 56.7\% cases showed lymph nodes metastasis(Schneider et al., 2018).

The mean age of patients recorded was $59.5 \pm 13.63$ (mean $\pm \mathrm{SD}$ ) with least age as 34 years and the extreme as 91 years. Whereas higher incidence of HNSCC was seen in 40 years and above age groups in the studies conducted in Norway, Sweden, Denmark and Finland in (Akram, Mirza, Mirza, \& Qureshi, 2013). However, some of the previous studies that are being conducted in Pakistan and India show the same results. Similarly, the age distribution in our study showed that 37 out of 64 patients were of the age below 60 years and 27 were of age above 60 years.

Among the patients $44(66.7 \%)$ were males and $22(33.3 \%)$ were females along with a male: female proportion of $2: 1$, that showed a consistency with the local and international previous studies. Whereas inconsistent with a metanalysis carried out in 2019 in Spain showing $56 \%$ female and $44 \%$ male patients and favoring the high PD- L1 expression in female patients.(Lenouvel et al., 2020)

In the current study, the most common site for HNSCC was mandible $(37.9 \%)$ followed by maxilla $25.8 \%$, buccal mucosa $13.6 \%$, tongue $10.6 \%$, Palate $4.5 \%$, floor of the mouth $4.5 \%$ and salivary glands $3 \%$. However in Asia the buccal mucosa is known to be most commonly affected area followed by the tongue(Sharma, Saxena, \& Aggarwal, 2010). which might be because of oral habits of the local population.

According to the histological grading of HNSCC there were 22(33.3\%) well differentiated SCC cases, 28(42.4\%) moderately differentiated SCC cases, $15(22.7 \%)$ poorly differentiated SCC cases and grade of one case couldn't be assessed. Which was in contrast to the study carried out in 2020 in which $87 \%$ cases were of poorly differentiated Squamous Cell Carcinoma (Lenouvel et al., 2020)

In this study PD - 1 and PD - L1 IHC expression was assessed in primary tumor sections and corresponding lymph nodes that were metastasized. In total of 66 cases, $47(71.2 \%)$ cases showed positive expression of PD - 1 in the tumor infiltrating lymphocytes seenin theprimary tumor sections of the specimens, whereas 40 (60.6\%) cases presented PD-1 positivity in cancer cells of primary tumor. These results are parallel to international studies which concluded that the PD - L1 positive cases showed from $7 \%$ to $87 \%$ positivity (Lenouvel et al., 2020).In 
a study, PD - 1 and PD-L1 targeting immunotherapy for metastatic and also recurrent HNSCC has shown improvement in patient survival (Ferris et al., 2018), furthermore better overall survival rate is seen in tumors expressing high PD-L1 levels (Cohen et al., 2019).

The PD - L1 expression is more commonly seen in the tumors with increased number of lymphocytes, however the p-value was insignificant. A study conducted in 2019 stated a relation among the PD-L1 overexpression showing increased number of TILs (Khan et al., 2019). This shows that malignant cells use the immune system for its own advantage (Lenouvel et al., 2020).

In our study another significant association was seen amongst PD - L1 expression in primary tumor besides pathological staging of regional lymph nodes $(p=0.04)$. which was different from the study carried out in 2020 showing insignificant relation between the $\mathrm{n}$ stage of tumor and PD - L1 expression (Gennen et al., 2020).

Surprisingly a lower PD - L1 expression was seen in high-grade OSCC than that in a low-grade OSCC and these data contradicted previous study carried out in 2017 (Hirai et al., 2017). A further investigation is though suggested to test this hypothesis.

In this study PD - 1 positivity was seen in 39 out of 46 (84.8\%) cases with lymph node metastasis. It presented a noteworthy relation between the PD - 1 expression of TIL $\mathrm{s}$ in primary tumor and lymph nodes metastasis $(\mathrm{p}=0.003)$. Similarly in a study conducted in Spain PD - 1 expression in TIL $\mathrm{s}$ linked significantly in corresponding primary tumor and metastasis in lymph-nodes $(\mathrm{p}=0.0412), \mathrm{PD}-1$ expressing lymphocytes were found in the 90\% (69/77) samples of lymph nodes (Schneider et al., 2018).

In our study another significant association was seen among the expression of PD - 1 in TIL $\mathrm{s}$ in tumor proper sections and expression of PD-1 in the associated metastatic lymph nodes (38/46 cases were positive with $\mathrm{p}=0.003$ )

In total of 66 cases, 48 cases were associated with lymph nodes metastasis and a significant association $(p=0.027)$ was seen among the PD - L1 expression and lymph nodes metastasis as $67.4 \%$ (33/48) showed positive PD - L1 expression in primary tumor. In a study conducted in 2019 it was concluded that high immune histochemicalexpression of PD - L1 might be a significant factor in prediction anti - PD - 1/PD - L1 monotherapy efficacy (Xu et al., 2019).

Despite the fact that conventionally chemical and radical therapies have been in use for the treatment of the cancer, still immunotherapy is considered to be the most effective against the cancers (Longo et al., 2019). The most integral suppressors of cytotoxic immune reaction considered are PD - 1 and PD - L1, and their antibody has been approved by FDA (Force et al., 2019).

The studies conducted in 2019 concluded that high PD - 1 expression was linked with the improved subsistence of the breast carcinomas patients (Jiang et al., 2019) and patients showing high PD-L1 expressions are expected to get more benefit from the antiPD-1/PD-L1(Xu et al., 2019).

The main limitation if this study was sample size. It was relatively smaller for such kind of epidemiological studies. A larger sample size could additionally increase validation of the results of the study. The further recommendation is to conduct the study on relatively larger sample size. This study has paved the path for further research in the field of immunotherapy in HNSCC. However, the conclusions of the study sustenance more studies to further prove the use of immune checkpoint inhibitors in the therapy of HNSCC.

Conclusively, the vital part of checkpoint (PD-1 and PD-L1) inhibitors in most cancers makes them to be researched further as they act an opportunity as well as a challenge for cancer treatment. A variety of solid cancers show increased IHC expression of PD- 1 and PD-L1. Immunotherapy being a promising and advantageous treatment seems to be an important step in the cancer therapy. PD-1 expressing TILs and PD-L1 in primary tumor cells of primary tumor along with corresponding lymph nodes showed an association with the cancer profiles.

In the relevance of PD -L1 and PD - 1 expression, the study done provides helpful data for the new treatment regimens as the results of our study further emphasizes on the expression of PD -1 and PD -L1 in HNSCC and their significant association with nodal metastasis seen.

Appropriate and accurate assessment with the diagnosis and successful treatment with the help of these IHC markers would lead to the early and timely diagnosis followed by a positive treatment outcome. The expression of PD-L1 in tumor cells and PD-1 in tumor infiltrating lymphocytes may help the clinician and oncologist in many ways, like better delineation of treatment regimens along with the prognosis of the disease. Also, it will help in the appropriate assessment of the course of disease also at time of the initial diagnosis on incisional biopsies.

\section{Author Contribution Statement}

IqraaShakeel Malik: Interpretaion of IHC staining and menuscript writing. Muhammad Asif: Study conception and design along with histopathological diagnosis and interpretation. Namrah Bashir : Data Collection. Nighat Ara : Menuscript writing. Farhat Rashid : Review of IHC results. Hafeez Ud Din: Data Analysis. Numrah Shakeel Malik : Menuscript writing. Aimen Bashir : Data Collection

\section{Acknowledgements}

I would express my appreciation for my Institute, Armed Forces Institute of Pathology (AFIP) for providing the excellent research environment and facilities. I am very thankful to the entire staff of Pathology laboratory, AFIP, Rawalpindi who helped me relentlessly from day one.

\section{Source of Funding}

No external source of funding was involved in this research. It was partly borne by the institute, National University of Medical Sciences, Rawalpindi and partly by the researcher.

Asian Pacific Journal of Cancer Prevention, Vol 23 


\section{Institutional Review Board Approval}

The armed forces institute of pathology conducted a institutional review board committee meeting for the approval of synopsis. It was approved via letter: MP-ORP18-6/READ-IRB/19/645.

\section{Ethical review board committee approval}

IRB approval was given by the Ethical Review Committee AFIP via letter: MP-ORP18-6/READIRB/19/645. A signed and telephonic consent was obtained from all participants in the study.

\section{Conflict of Interest}

No conflict of interest was declared in this study.

\section{References}

Akram S, Mirza T, Mirza MA, Qureshi M (2013). Emerging patterns in clinico-pathological spectrum of oral cancers. Pak J Med Sci, 29, 783.

Al Azhar M, Aisyi M (2021). Profile of PD-L1 mRNA expression in childhood acute leukemia. Asian Pac J Cancer Biol, 6, $37-41$.

Alsaab HO, Sau S, Alzhrani R, et al (2017). PD-1 and PD-L1 checkpoint signaling inhibition for cancer immunotherapy: mechanism, combinations, and clinical outcome. Front Pharmacol, 8, 561.

Brahmer JR, Tykodi SS, Chow LQ, et al (2012). Safety and activity of anti-PD-L1 antibody in patients with advanced cancer. N Engl J Med, 366, 2455-65.

Chemnitz JM, Parry RV, Nichols KE, June CH, Riley JL (2004). SHP-1 and SHP-2 associate with immunoreceptor tyrosinebased switch motif of programmed death 1 upon primary human $\mathrm{T}$ cell stimulation, but only receptor ligation prevents T cell activation. $J$ Immunol, 173, 945-54.

Chen L, Han X (2015). Anti-PD-1/PD-L1 therapy of human cancer: past, present, and future. J Clin Invest, 125, 3384-91.

Cohen EE, Soulières D, Le Tourneau C, et al (2019). Pembrolizumab versus methotrexate, docetaxel, or cetuximab for recurrent or metastatic head-and-neck squamous cell carcinoma (KEYNOTE-040): a randomised, open-label, phase 3 study. Lancet, 393, 156-67.

Elmusrati A, Wang J, Wang C-Y (2021). Tumor microenvironment and immune evasion in head and neck squamous cell carcinoma. Int J Oral Sci, 13, 24.

Ferris RL, Blumenschein Jr, G, Fayette J, et al (2018). Nivolumab vs investigator's choice in recurrent or metastatic squamous cell carcinoma of the head and neck: 2-year long-term survival update of CheckMate 141 with analyses by tumor PD-L1 expression. Oral Oncol, 81, 45-51.

Force J, Leal JHS, McArthur HL (2019). Checkpoint blockade strategies in the treatment of breast cancer: where we are and where we are heading. Curr Treat Options Oncol, 20, 1-14.

Gennen K, Käsmann L, Taugner J, et al (2020). Prognostic value of PD-L1 expression on tumor cells combined with CD8+ TIL density in patients with locally advanced non-small cell lung cancer treated with concurrent chemoradiotherapy. Radiat Oncol, 15, 1-12.

Goel H, Singhal S, Mathur R, et al (2020). Promoter Hypermethylation of LATS2 Gene in Oral Squamous Cell Carcinoma (OSCC) among North Indian Population. Asian Pac J Cancer Prev, 21, 1283.

Gorsky M, Epstein JB, Oakley C, et al (2004). Carcinoma of the tongue: a case series analysis of clinical presentation, risk factors, staging, and outcome. Oral Surg Oral Med Oral Pathol Oral Radiol Endodontol, 98, 546-52.
Hanna E (2020). How fragile we are. Head Neck, 42, 1355.

Hirai M, Kitahara H, Kobayashi Y,et al (2017). Regulation of PD-L1 expression in a high-grade invasive human oral squamous cell carcinoma microenvironment. IntJ Oncol, 50, $41-8$.

Jiang C, Cao S, Li N, Jiang L, Sun T (2019). PD-1 and PD-L1 correlated gene expression profiles and their association with clinical outcomes of breast cancer. Cancer Cell Int, 19, 1-9.

Khan MA, Syed S, Wahab NU, Butt SA (2019). Immunohistochemical expression of programmed death ligand-1 in tumour Milieu of oral cancer. Annals of Abbasi Shaheed Hospital and Karachi Medical \& Dental College, 24, pp 187-94.

Lenouvel D, González-Moles MÁ, Ruiz-Ávila I, et al (2020). Prognostic and clinicopathological significance of PD-L1 overexpression in oral squamous cell carcinoma: A systematic review and comprehensive meta-analysis. Oral Oncol, 106, 104722.

Lin Y-M, Sung W-W, Hsieh M-J, et al (2015). High PD-L1 expression correlates with metastasis and poor prognosis in oral squamous cell carcinoma. PLoS One, 10, e 0142656.

Longo V, Brunetti O, Azzariti A, et al (2019). Strategies to improve cancer immune checkpoint inhibitors efficacy, other than abscopal effect: A systematic review. Cancers, 11, 539.

Maruse Y, Kawano S, Jinno T, et al (2018). Significant association of increased PD-L1 and PD-1 expression with nodal metastasis and a poor prognosis in oral squamous cell carcinoma. Int J Oral Maxillofacial Surg, 47, 836-45.

Pardoll DM (2012). The blockade of immune checkpoints in cancer immunotherapy. Nat Rev Cancer, 12, 252-64.

Patil S, Rao R, Amrutha N, Sanketh D (2014). Analysis of human papilloma virus in oral squamous cell carcinoma using p16: An immunohistochemical study. J Int Soc Prev Commun Dentist, 4, 61.

Schneider S, Kadletz L, Wiebringhaus R, et al (2018). PD-1 and PD-L1 expression in HNSCC primary cancer and related lymph node metastasis-impact on clinical outcome. Histopathology, 73, 573-84.

Schreiber RD, Old LJ, Smyth MJ (2011). Cancer immunoediting: integrating immunity's roles in cancer suppression and promotion. Science, 331, 1565-70.

Sharma P, Saxena S, Aggarwal P (2010). Trends in the epidemiology of oral squamous cell carcinoma in Western UP: an institutional study. Indian J Dent Res, 21, 316.

Syn NL, Teng MW, Mok TS, Soo RA (2017). De-novo and acquired resistance to immune checkpoint targeting. Lancet Oncol, 18, 731-41.

Teixidó C, Vilariño N, Reyes R, Reguart N (2018). PD-L1 expression testing in non-small cell lung cancer. Ther $A d v$ Med Oncol, 10, 1758835918763493.

Wang Y, Wang J, Huang Y (2015). MicroRNAs as new biomarkers for human papilloma virus related head and neck cancers. Cancer Biomarkers, 15, 213-8.

Wu Y-S, Lin P-Y, Chien C-Y, et al (2016). Anxiety and depression in patients with head and neck cancer: 6-month follow-up study. Neuropsychiatric Dis Treat, 12, 1029.

Xu Y, Wan B, Chen X, et al (2019). The association of PDL1 expression with the efficacy of anti-PD-1/PD-L1 immunotherapy and survival of non-small cell lung cancer patients: a meta-analysis of randomized controlled trials. Transl Lung Cancer Res, 8, 413.

Zeng S, Fu L, Zhou P, Ling H (2020). Identifying risk factors for the prognosis of head and neck cutaneous squamous cell carcinoma: A systematic review and meta-analysis. PLoS One, 15, e0239586. 
DOI: $10.31557 /$ APJCP.2022.23.2.467

Immunohistochemical Expression Of PD-1, PD-L1 And Its Association With Nodal Metastasis

\section{(ब) $(\mathbb{8}$}

This work is licensed under a Creative Commons Attribution-

Non Commercial 4.0 International License. 